\title{
Efeitos relativísticos sobre observadores uniformemente acelerados
}

\author{
Relativistic effects on uniformly accelerated observers \\ Reinaldo da Silva Caraça*1 \\ ${ }^{1}$ Instituto Federal de Educação, Ciência e Tecnologia de São Paulo - Câmpus São Paulo Pirituba, Av. Mutinga, 951, Jardim \\ Santo Elias, São Paulo, SP, Brasil
}

Recebido em 03 de Janeiro de 2017. Aceito em 26 de Fevereiro de 2017

\begin{abstract}
Neste trabalho apresentaremos uma introdução sobre o espaco-tempo de Rindler a partir de uma abordagem didática a fim de obtermos as equações de movimento que descrevem observadores uniformemente acelerados em um espaco-tempo plano. Em seguida, generalizaremos, descrevendo o movimento associado a um observador uniformemente acelerado no espaco-tempo em expansão de Friedmann-Lemaître-Robertson-Walker.

Palavras-chave: relatividade especial; espaço-tempo de Rindler; 4-aceleração; espaço-tempo em expansão.
\end{abstract}

In this article we presented an introduction to Rindler spacetime from a didactic approach in order to get the motion equations describing uniformly accelerated observers in the smooth spacetime. Then, we will treat the motion of uniformely accelerated observer in Friedmann-Lemaitrre-Robertson-Walker expanding spacetime.

Keywords: special relativity; Rindler spacetime; 4-acceleration; expanding spacetime.

\section{Introdução}

Na década de 1960, W. Rindler pôs-se, dentre outras coisas, a estudar o movimento descrito por um observador uniformemente acelerado no espaco-tempo de Minkowski 1].

Os resultados obtidos são amplamente conhecidos e servem de base, por exemplo, na formulacão do efeito FullingDavies-Unruh [2 4], segundo o qual para um observador acelerado, o vácuo de Minkowski não é o estado de menor energia mas, ao invés disso, é percebido como um banho térmico ao qual se associa uma temperatura (temperatura Unruh), proporcional a aceleração do observador. Uma apresentação concisa e didática sobre o fenômeno pode ser conferida na referência [5].

Embora o espaço-tempo de Rindler seja apresentado na maioria dos bons livros-texto de relatividade [6 9], detalhes quase sempre são omitidos o que resulta em uma certa confusão por parte dos estudantes quanto a sua interpretação.

Este trabalho está organizado da seguinte forma: na seção 2, faremos uma revisão sobre o espaço-tempo de Minkowski, apresentando suas características, além de conceitos como tempo-próprio e diferentes tipos de intervalos espaço-temporais. Em seguida, tratar-se-á do movimento uniformemente acelerado (hiperbólico) no espaço-tempo de Rindler, destacando algumas de suas características principais e culminando com a comparação entre o movimento uniformemente acelerado relativístico

*Endereço de correspondência: reinaldo.caraca@ifsp.edu.br e o clássico. Já na seção 3, estenderemos o estudo do movimento de observadores acelerados para o caso de um universo plano em expansão. Finalmente, na seção 4 apresentaremos nossas conclusões.

\section{Espaço-tempo de Minkowski}

Geometricamente, a principal diferença entre a física clássica e a relativística é que o espaço e o tempo, absolutos, distintos e não interagentes com os eventos, segundo a perspectiva clássica 10 passam a ser tratados como equivalentes e inseparáveis na concepção relativística. Aqui, não se pode falar em espaço dissociado do tempo mas sim em um "entrelaçado" espaço-tempo.

A teoria de Einstein teve de "sacrificar" a característica absoluta entre tempo e espaço e transferi-la à velocidade da luz, passando a interagirem diretamente com eventos. Como consequência, a noção de simultaneidade torna-se relativa: dois eventos podem ser simultâneos para um observador e não serem para outro.

Outra grandeza, além da velocidade da luz, que mantém seu significado absoluto na teoria relativística é o elemento de linha, ou linha de mundo, que fisicamente nos diz como generalizar a noção de distância na teoria relativística, denotado por: $d s^{2}$.

Para um espaço-tempo plano, isto é, na ausência de gravitação, temos o seguinte elemento de linha (em coordenadas cartesianas):

$$
d s^{2}=c^{2} d t^{2}-d x^{2}-d y^{2}-d z^{2},
$$


onde $c$ é a velocidade da luz, $t$ é a coordenada temporal que, em relatividade, é chamada de coordenada tipotempo, ao passo que $x, y, z$ são coordenadas tipo-espaço. A equação (1) apresenta diferenças sensíveis em relação à geometria Euclidiana. Primeiramente, nota-se a presença de sinais negativos (na geometria euclidiana a distância é uma grandeza invariante formadas pela soma dos quadrados dos elementos). Depois, verifica-se o aparecimento da coordenada temporal juntamente com as espaciais, o que não ocorrem na geometria de Euclides e dá origem ao espaço-tempo descrito pelo elemento de linha (1), chamado espaço-tempo de Minkowski.

A teoria relativística pode ser formulada consistentemente em termos de tensores, como pode ser verificado em praticamente todos os livros sobre o assunto $7,8,11,13$. Isto compactifica a notação, simplificando-a graficamente e tornando-a matematicamente elegante. A equação (1), por exemplo, pode ser reescrita em notação tensorial como

$$
d s^{2}=g_{\mu \nu} d x^{\mu} d x^{\nu},
$$

sendo $g_{\mu \nu}$ o tensor métrico que, no caso de Minkowski, em coordenadas cartesianas, é dado pela matriz diagonal $g=\operatorname{diag}(1,-1,-1,-1)$. Onde os índices gregos, seguindo a notação utilizada neste trabalho, variam desde 0 até 3 , e $d x^{0} \equiv c^{2} d t^{2}, d x^{1} \equiv d x, d x^{2} \equiv d y$ e $d x^{3} \equiv d z$.

Além disso, usaremos a chamada convenção de Einstein segundo a qual, índices repetidos, estando um embaixo e outro em cima, representam soma

$$
F_{\alpha \beta} x^{\alpha} \equiv \sum_{\alpha} F_{\alpha \beta} x^{\alpha}
$$

assim

$$
\begin{aligned}
d s^{2} & =g_{\mu \nu} d x^{\mu} d x^{\nu}=\sum_{\mu, \nu} g_{\mu \nu} d x^{\mu} d x^{\nu} \\
& =g_{00} d x^{0} d x^{0}+g_{01} d x^{0} d x^{1}+\ldots+g_{33} d x^{3} d x^{3}(3)
\end{aligned}
$$

\subsection{Tempo-próprio}

Conforme já dissemos, na física clássica o tempo possui uma natureza absoluta, o que não ocorre na relatividade. Assim, o tempo medido por quem está em movimento e carrega consigo o relógio é diferente daquele tempo medido por um observador inercial em repouso e que vê o movimento ocorrer com velocidade $v$ em relação a si. Ambos intervalos de tempo medidos pelos diferentes relógios estão relacionados pela seguinte transformação de Lorentz [13]:

$$
\Delta t=\frac{1}{\sqrt{1-(v / c)^{2}}}\left(\Delta \bar{t}+\frac{v \Delta \bar{x}}{c^{2}}\right),
$$

onde as coordenadas com "barra" se referem a um observador atrelado ao sistema em movimento. Para ele, sua posição não varia e seu relógio se encontra em repouso relativo a $\bar{S}$. O intervalo de tempo medido por este relógio é denominado tempo-próprio $(\tau)$ e, como veremos é uma grandeza invariante, isto é, todos os observadores devem concordar quanto a sua medida.

Um observador fixo em S, por sua vez, vê $\bar{S}$ passar com velocidade $v$ (conforme mostrado na Figura (1)) e o intervalo de tempo decorrido entre dois eventos distintos e medido por este observador é denotado por $\Delta t$.

Então, a partir da transformação (4)

$$
\Delta \bar{t}=\Delta \tau=\sqrt{1-(v / c)^{2}} \Delta t .
$$

Perceba que o intervalo de tempo-próprio, $\Delta \tau$, é sempre menor que o intervalo de tempo medido por um observador inercial, cujo relógio se move relativamente a $\bar{S}$.

Como o elemento de linha (1) é invariante, podemos igualar $d s^{2}$ referente ao observador fixo no referencial $\mathrm{S}$ com $\overline{d s}^{2}$ de outro observador, em repouso com relação a $\bar{S}$ :

$$
\begin{aligned}
& (c \overline{d t})^{2}-(\overline{d x})^{2}-(\overline{d y})^{2}-(\overline{d z})^{2} \\
= & (c d t)^{2}-(d x)^{2}-(d y)^{2}-(d z)^{2},
\end{aligned}
$$

Para o observador fixo em $\bar{S}$, suas coordenadas espaciais não se alterarão, o que transforma $(6) \mathrm{em}$

$$
(c d \tau)^{2}=(c d t)^{2}-(d x)^{2}-(d y)^{2}-(d z)^{2}=d s^{2} .
$$

Conclui-se, portanto, que o tempo-próprio é uma quantidade invariante, independente do referencial.

\subsection{Tipos de intervalos}

Para o elemento de linha descrito pela equação (1) temos três possibilidades:

$1^{\mathrm{o}}(d s)^{2}>0$ : Intervalo tipo-tempo.

Caracterizado por $(c d t)^{2}>g_{i j} d x^{i} d x^{j}(i=1,2,3)$. Dividindo todos os termos por $(d t)^{2}$ temos que $\left(v_{x}\right)^{2}+\left(v_{y}\right)^{2}+\left(v_{z}\right)^{2}=(v)^{2}<c^{2}$. Este é o tipo de intervalo que descreve o movimento de partículas físicas:

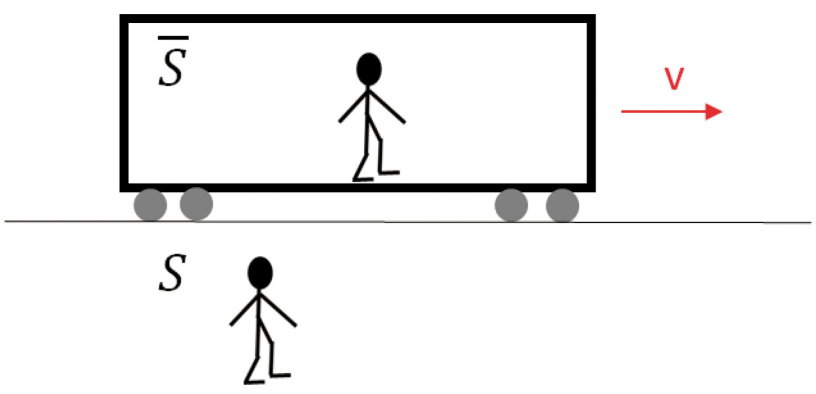

Figura 1: O observador em $\bar{S}$ encontra-se em repouso em relação ao vagão, desta forma, o intervalo de tempo medido por ele será igual ao tempo-próprio. Por outro lado, o observador situado às margens da estrada, verá o vagão se movendo em relação ao seu relógio, registrando um intervalo de tempo $\Delta t$ maior que aquele medido em $\bar{S}$. 
$2^{\mathrm{o}}(d s)^{2}<0$ : Intervalo tipo-espaço.

Aqui, $(c d t)^{2}<g_{i j} d x^{i} d x^{j}(i=1,2,3)$, ou seja, implica velocidades maiores que a da luz;

$3^{\text {o }}(d s)^{2}=0$ : Intevalo tipo-luz.

Agora $(c d t)^{2}=g_{i j} d x^{i} d x^{j}$, ou seja, $v=c$. Descreve trajetórias de raios de luz.

Desta forma, o espaço-tempo é dividido em três regiões distintas: uma tipo-tempo, outra tipo-espaço e, separando ambas, a tipo-luz. A representação destas é vista na Figura 20.

\subsection{Movimento hiperbólico no espaço-tempo de Minkowski}

A cada partícula, em relatividade, associamos um 4-vetor $u^{\mu}$ denominado 4-velocidade, definido como

$$
u^{\mu} \equiv \frac{d x^{\mu}}{d \tau}
$$

onde $x^{\mu}=(c t, x, y, z)$ em um sistema de coordenadas cartesianas retangulares.

A partir da 4-velocidade define-se a 4-aceleração:

$$
a^{\mu} \equiv \frac{d u^{\mu}}{d \tau}=\frac{d^{2} x^{\mu}}{d \tau^{2}} .
$$

Como já vimos, a relação entre os tempos do observador em repouso $d t$ e aquele no referencial da partícula $d \bar{t}$ é obtida mediante transformações de Lorentz

$$
\begin{aligned}
d t & =\frac{1}{\sqrt{1-(v / c)^{2}}} d \bar{t} \\
\frac{d t}{d \bar{t}} & =\frac{d t}{d \tau}=\gamma \equiv \frac{1}{\sqrt{1-(v / c)^{2}}} .
\end{aligned}
$$

Com isto, as expressões (8) e t9rnam-se

$$
\begin{aligned}
& u^{\mu}=\frac{d x^{\mu}}{d t} \frac{d t}{d \tau}=\gamma(c, \mathbf{v}), \\
& a^{\mu}=\frac{d u^{\mu}}{d t} \frac{d t}{d \tau}=\gamma[(c \dot{\gamma}, \dot{\gamma} \mathbf{v}+\gamma \dot{\mathbf{v}})]
\end{aligned}
$$

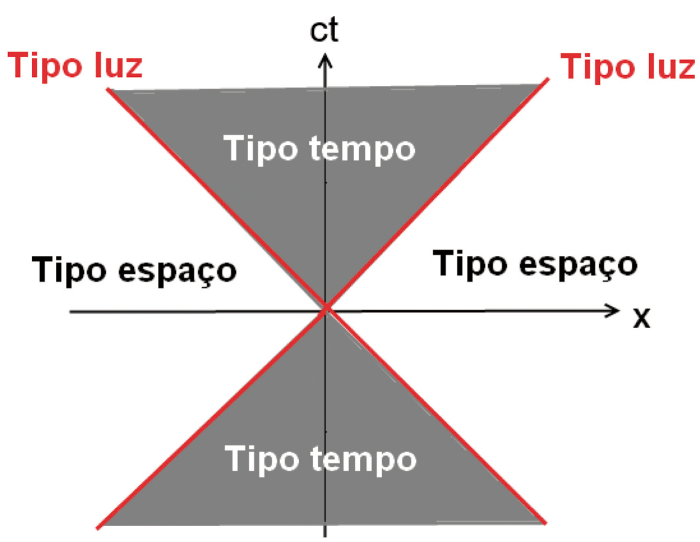

Figura 2: Partículas massivas estão restritas à região interna ao cone (tipo-tempo). A região externa ao cone, tipo espaço, está causalmente desconectada do interior e a fronteira entre ambas forma o intervalo tipo-luz, que descreve linhas de mundo de raios de luz onde o "ponto" corresponde à derivada em relação à coordenadada tipo-tempo $t$.

Tomemos agora o valor do invariante relacionado com a 4-aceleração $g_{\mu \nu} a^{\mu} a^{\nu}$ :

$$
\begin{aligned}
g_{\mu \nu} a^{\mu} a^{\nu} & =\gamma^{2}\left[c^{2} \dot{\gamma}^{2}-\dot{\gamma}^{2} v^{2}-2 \gamma \dot{\gamma} \mathbf{v} \cdot \dot{\mathbf{v}}-\gamma^{2} \dot{\mathbf{v}} \cdot \dot{\mathbf{v}}\right] \\
& =\gamma^{6} \dot{v}^{2} \frac{v^{2}}{c^{2}}\left(1-(v / c)^{2}\right)-2 \gamma^{6} \frac{v^{2}}{c^{2}} \dot{v}^{2}-\gamma^{4} \dot{\mathbf{v}}^{2}, \\
& =-\gamma^{6} \frac{v^{2}}{c^{2}} \dot{v}^{2}-\gamma^{4} \dot{\mathbf{v}}^{2} .
\end{aligned}
$$

Se o movimento não ocorrer sobre uma linha reta, $\|\dot{\mathbf{v}}\| \neq$ $\dot{v}$, isto pode ser facilmente visto no caso de um movimento circular uniforme, nele o módulo da velocidade não se altera, de modo que $\dot{v}=0$. Entretanto, $\dot{\mathbf{v}} \neq 0$ já que, mesmo não havendo variação na intensidade da velocidade, esta varia em direção.

Por outro lado, se o movimento for retilíneo, ambas derivadas coincidirão e

$$
\begin{aligned}
g_{\mu \nu} a^{\mu} a^{\nu} & =-\gamma^{6} \frac{v^{2}}{c^{2}} \dot{v}^{2}-\gamma^{4} \dot{v}^{2}=-(\alpha)^{2} \\
& =\gamma^{4} \dot{v}^{2}\left(\frac{(v / c)^{2}}{1-(v / c)^{2}}+1\right)=-(\alpha)^{2} \\
\gamma^{6} \dot{v}^{2} & =(\alpha)^{2} .
\end{aligned}
$$

Até agora, este resultado é geral, ou seja, é válido para qualquer referencial e $\alpha$ é uma função da posição e do tempo.

Adotemos aqui a particularidade escolhendo $\alpha=$ cte ao longo do eixo x positivo. Assumindo ainda que em $t=0$ a partícula se encontrava em repouso momentâneo, obtemos

$$
\gamma^{3} \frac{d v}{d t}=\frac{d}{d t}(\gamma v)=\alpha \Rightarrow t=\frac{\gamma v}{\alpha} .
$$

Elevando a última igualdade ao quadrado e isolando a velocidade, tiramos a expressão para $x(t)$

$v=\frac{d x}{d t}=\frac{\alpha t}{\sqrt{1+(\alpha t / c)^{2}}} \Rightarrow x(t)=\frac{c^{2}}{\alpha} \sqrt{1+\frac{\alpha^{2} t^{2}}{c^{2}}}+b_{1}$.

Podemos escolher $b_{1}=0$, de modo que

$$
x^{2}=\frac{c^{4}}{\alpha^{2}}+c^{2} t^{2} \Rightarrow x^{2}-c^{2} t^{2}=\frac{c^{4}}{\alpha^{2}} .
$$

A equação 15 pode ser parametrizada em termos de funções hiperbólicas como

$$
\begin{aligned}
x & =\rho \cosh (\beta \eta), \\
c t & =\rho \sinh (\beta \eta),
\end{aligned}
$$

onde $\beta$ é uma constante.

Ao substituírmos as equações anteriores em (15) concluímos que $\rho=c^{2} / \alpha$. 
Os outros parâmetros são obtidos a partir do elemento de linha:

$$
\begin{aligned}
(d s)^{2} & =(c d \tau)^{2}=(c d t)^{2}-(d x)^{2}-(d y)^{2}-(d z)^{2} \\
& =\frac{\beta^{2} c^{4}}{\alpha^{2}}(d \eta)^{2}-(d y)^{2}-(d z)^{2}
\end{aligned}
$$

donde tiramos $\beta=\alpha / c^{2}$ e $\eta=c \tau$, portanto,

$$
\begin{aligned}
x(\tau) & =\frac{c^{2}}{\alpha} \cosh (\alpha \tau / c), \\
t(\tau) & =\frac{c}{\alpha} \sinh (\alpha \tau / c) .
\end{aligned}
$$

O diagrama para o espaço-tempo de Rindler sob as transformações acima é mostrado na figura (3).

A interpretação física do movimento descrito por uma partícula de Rindler é a seguinte. Considere uma partícula sujeita a uma aceleração constante ao longo de $x$ positivo. Em $t \rightarrow-\infty$ a partícula possui velocidade uma velocidade inicial no sentido negativo do eixo $x$, à medida que o tempo aumenta a partícula aproxima-se de $\mathrm{x}=0$, sendo freada a uma taxa constante. Ao atingir $x=c^{2} / \alpha$ a velocidade da partícula se anula e, a partir daí, ela passa a ser acelerada no sentido de $x$ positivo, voltando para o infinito.

Ainda com relação à figura (3), podemos ver que um observador acelerado não tem acesso a todo o espaçotempo. A região do quarto quadrante, situada abaixo da reta $x=-c t$, pode emitir sinal detectável pelo observador acelerado, descrito pela linha-mundo hiperbólica, contudo, este não pode enviar sinal para este setor do espaço-tempo.

Por outro lado, a região do primeiro quadrante, localizada acima de $x=$ ct pode receber sinal do observador

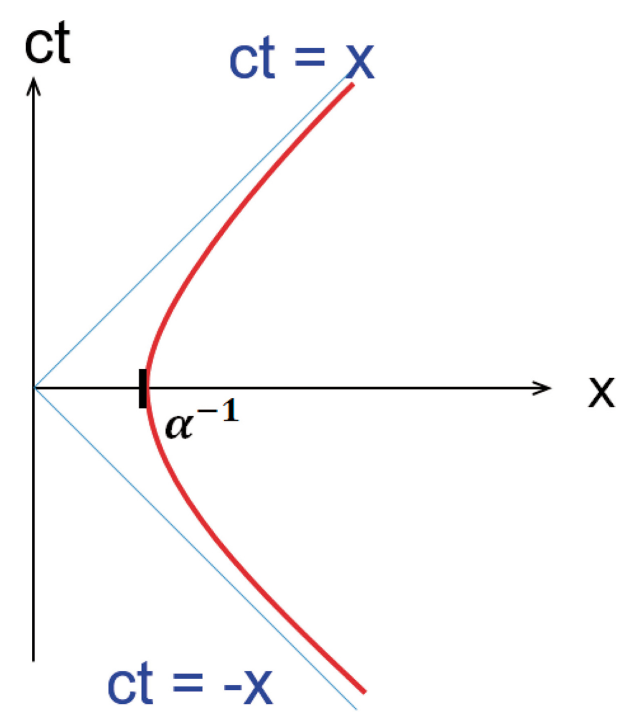

Figura 3: Diagrama de espaço-tempo para observadores de Rindler. Veja que quando $t$ torna-se muito grande, as trajetórias se aproximam mais e mais das retas $c t=x$ e $c t=-x$ nunca atingindo-as entretanto. acelerado mas não consegue se comunicar com ele.

Ambas regiões são análogas ao horizonte de eventos dos buracos negros, entretanto, enquanto o horizonte de eventos é uma estrutura intrínseca ao espaço tempo, o horizonte de Rindler depende claramente do movimento do observador.

\subsubsection{Outra dedução das funções do movimento}

O procedimento descrito na subseção anterior é o que foi utilizado por Rindler em seu artigo de 1960 [1] onde, a partir da integração da aceleração, se obtém uma relação envolvendo a coordenada tipo-tempo e a velocidade que, novamente integrada, fornece a coordenada tipo-espaço em função da coordenada tipo-tempo. Finalmente, usando o elemento de linha, os relaciona com o tempo-próprio.

Um outro procedimento para obtermos as mesmas expressões para $t(\tau)$ e $x(\tau)$ é descrito a partir de agora.

Comecemos utilizando a expressão para o elemento de linha em Minkowski, uma vez que queremos estudar o movimento de um observador acelerado em um espaçotempo plano.

$$
\begin{aligned}
d s^{2} & =c^{2} d \tau^{2}=(c d t)^{2}-(d x)^{2} \Rightarrow \\
1 & =\left(\frac{d t}{d \tau}\right)^{2}-\left(\frac{d x}{c d \tau}\right)^{2} \\
& \Rightarrow\left(c t^{\prime}\right)^{2}-\left(x^{\prime}\right)^{2}=c^{2} .
\end{aligned}
$$

A partir de agora, por simplicidade de notação, denotaremos derivadas com relação ao tempo-próprio por "linhas".

Por outro lado, um observador uniformemente acelerado (com 4-aceleração constante) satisfaz ainda

$$
\begin{aligned}
g_{\mu \nu} a^{\mu} a^{\nu} & =-\alpha^{2}=c t e \\
g_{00}\left(a^{0}\right)^{2}+g_{11}\left(a^{1}\right)^{2} & =\left(c t^{\prime \prime}\right)^{2}-\left(x^{\prime \prime}\right)^{2}=-\alpha^{2} .
\end{aligned}
$$

Isolando $x^{\prime}$ em 21 derivando-a mais uma vez com relação ao tempo-próprio

$$
x^{\prime} x^{\prime \prime}=c^{2} t^{\prime} t^{\prime \prime} \Rightarrow x^{\prime \prime}=c^{2} \frac{t^{\prime} t^{\prime \prime}}{x^{\prime}}=c \frac{t^{\prime} t^{\prime \prime}}{\sqrt{t^{\prime 2}-1}} .
$$

Substituindo agora em 22 ficamos com a seguinte equação diferencial

$$
t^{\prime \prime}=\frac{\alpha}{c} \sqrt{t^{2}-1} .
$$

Esta equação diferencial pode ser posta numa forma mais simples utilizando uma transformação de variável apropriada. Observando o termo no interior da raiz quadrada, uma transformação sugestiva é

$$
t^{\prime}=\cosh z \text {. }
$$

Tal escolha também é apresentada no artigo original de Rindler, embora não para resolver o caso em Minkowski. 
Com esta substituição na equação diferencial ficamos com

$$
\begin{aligned}
z^{\prime} \sinh z & =\frac{\alpha}{c} \sqrt{\cosh ^{2} z-1}=\frac{\alpha}{c} \sinh z \\
z^{\prime} & =\frac{\alpha}{c} \Rightarrow z=\alpha \tau / c+c t e
\end{aligned}
$$

Voltando para a variável $t$ através da transformação inversa de (24),

$$
\begin{aligned}
\cosh ^{-1} t^{\prime} & =\alpha \tau / c+c t e \Rightarrow t^{\prime}=\cosh (\alpha \tau / c+c t e) \\
t(\tau) & =\frac{c}{\alpha} \sinh (\alpha \tau / c+c t e)+c t e 2 .
\end{aligned}
$$

É possível, sem perda de conteúdo físico, escolher cte $2=0$ e, se supusermos que $t(\tau=0)=0$, concluímos que

$$
t(\tau)=\frac{c}{\alpha} \sinh (\alpha \tau / c) .
$$

Com este resultado, podemos voltar à equação 21 e obter $x(\tau)$

$$
\begin{aligned}
x^{\prime} & =\sqrt{c^{2} t^{\prime 2}-c^{2}}=c \sinh (\alpha \tau / c) \\
x & =\frac{c^{2}}{\alpha} \cosh (\alpha \tau / c)+c t e 3, \\
x(\tau) & =\frac{c^{2}}{\alpha} \cosh (\alpha \tau / c),
\end{aligned}
$$

onde escolhemos $c_{3}=0$.

Veja que reobtemos as expressões $(19)$ e $(20)$.

\subsubsection{Uma terceira dedução: coordenadas do cone de luz}

Analisando as equações (21) e 22 vemos que ambas equações diferenciais sugerem uma parametrização hiperbólica (diferença de quadrados). Podemos reescrevêlas como

$$
\begin{aligned}
\left(c t^{\prime}-x^{\prime}\right)\left(c t^{\prime}+x^{\prime}\right) & =c^{2}, \\
\left(c t^{\prime \prime}-x^{\prime \prime}\right)\left(c t^{\prime \prime}+x^{\prime \prime}\right) & =-\alpha^{2} .
\end{aligned}
$$

Definindo-se as coordenadas do cone de luz $u(x, t) \equiv$ $c t-x$ e $v(x, t) \equiv c t+x$, os vínculos associados ao movimento relativístico uniformemente acelerado passam a ser descritos como

$$
\begin{aligned}
u^{\prime} v^{\prime} & =c^{2}, \\
u^{\prime \prime} v^{\prime \prime} & =-\alpha^{2},
\end{aligned}
$$

enquanto o elemento de linha,

$$
d s^{2}=d u d v .
$$

A partir dos vínculos 28 e 29 chega-se aos mesmos resultados da subseção anterior. Para isso, isolemos $u^{\prime}$ em (28), derivando-o em relação a $\tau$ e, posteriormente substituindo-o em 29. Este procedimento resulta em

$$
\begin{aligned}
\frac{v^{\prime \prime}}{v^{\prime}} & = \pm \frac{\alpha}{c} \Rightarrow \frac{d}{d \tau} \ln \left(v^{\prime}\right)= \pm \frac{\alpha}{c}, \\
v & = \pm \frac{c}{\alpha} C_{1} e^{ \pm \alpha \tau / c}+D_{1}
\end{aligned}
$$

onde $C_{1}$ e $D_{1}$ são constantes.

Vamos escolher o sinal positivo para a exponencial e a constante $D_{1}=0$. Assim,

$$
v(\tau)=\frac{c}{\alpha} C_{1} e^{\alpha \tau / c},
$$

Derivando 31 e substituindo em 28 , após integrarmos a equação resultante chega-se a

$$
u(\tau)=-\frac{c}{\alpha C_{1}} e^{-\alpha \tau / c}
$$

Aqui também desprezamos a constante de integração. Já $C_{1}$, é determinada a partir de condições iniciais. No caso, estamos impondo que quando $t=0 \rightarrow \tau=0$ e ainda que $x(0)=c^{2} / \alpha$. Ambas considerações conduzem a $C_{1}=1$, então

$$
\begin{aligned}
x & =\frac{1}{2}(v-u)=\frac{c^{2}}{2 \alpha}\left(e^{\alpha \tau / c}+e^{-\alpha \tau / c}\right) \\
& =\frac{c^{2}}{\alpha} \cosh (\alpha \tau / c), \\
t & =\frac{1}{2 c}(u+v)=\frac{c}{2 \alpha}\left(e^{\alpha \tau / c}-e^{-\alpha \tau / c}\right) \\
& =\frac{c}{\alpha} \sinh (\alpha \tau / c),
\end{aligned}
$$

que são, novamente, as mesmas expressões apresentadas em 19 e 20 .

\subsection{Relação entre o referencial inercial e o acelerado}

O observador acelerado possui um sistema de coordenadas atrelado a si (comóvel) a partir do qual se utiliza para descrever o movimento ao seu redor e medir distâncias e intervalos de tempo as quais denominaremos coordenadas aceleradas $\left(\xi^{0}, \xi^{1}\right)$. Como estamos assumindo uma aceleração ao longo do eixo $x$, as demais dimensões espaciais permanecem inalteradas sua presença não trará nenhum novo conteúdo físico, por este motivo optamos em não explicitá-las.

No referencial comóvel ao observador acelerado, sua posição não se alterará e podemos escolhê-la como $\xi^{1}=0$, logo, somente a coordenada tipo-tempo evolui e esta deve corresponder exatamente ao tempo-próprio multiplicado pela velocidade da luz, medido por este observador: $\xi^{0}=c \tau$.

É importante se estabelecer a relação entre os sistemas de coordenadas inercial e aquele atrelado ao observador acelerado e, como ponto de partida, adotaremos uma forma mais geral para a métrica em função das coordenadas aceleradas

$$
\begin{aligned}
d s^{2}= & A\left(\xi^{0}, \xi^{1}\right)\left(d \xi^{0}\right)^{2}+B\left(\xi^{0}, \xi^{1}\right) d \xi^{0} d \xi^{1}+ \\
& +C\left(\xi^{0}, \xi^{1}\right)\left(d \xi^{1}\right)^{2} .
\end{aligned}
$$

Entretanto, o fato do espaço-tempo de Rindler ser parte do espaço-tempo de Minkowski introduz simetrias que 
nos permitem simplificar a equação (33).

Sabemos que o espaço tempo de Minkowski possui vetor de Killing tipo-tempo que se traduz na estacionariedade ou mais explicitamente, na independência temporal das componentes do tensor métrico. Como estes vetores de Killing se associam a isometrias e estas, independem do sistema de coordenadas adotado, isto também ocorre com o espaço-tempo de Rindler.

Some-se ainda o fato do espaço-tempo de Minkowski ser estático, isto é, não possui termos cruzados envolvendo a coordenada tipo-tempo na métrica. Desta forma, a equação 33 simplifica-se em

$$
d s^{2}=A\left(\xi^{1}\right)\left[\left(d \xi^{0}\right)^{2}-\left(d \xi^{1}\right)^{2}\right] .
$$

Acima, a coordenada tipo-espaço foi redefinida: $C\left(\xi^{1}\right) d \xi^{1} \rightarrow A\left(\xi^{1}\right) d \xi^{1}$.

Seguindo o procedimento realizado anteriormente, utilizaremos coordenadas do cone de luz para o observador acelerado:

$$
\tilde{u}=\xi^{0}-\xi^{1}, \quad \tilde{v}=\xi^{0}+\xi^{1} .
$$

Introduzamos um referencial comóvel e fixo no observador, $\xi^{1}=0$ e $\xi^{0}=c \tau$, de onde se conclui que

$$
\begin{aligned}
d s^{2} & =A\left(\xi^{1}\right)\left[(d \tau)^{2}\right] \\
d \tau^{2} & =A\left(\xi^{1}\right) d \tau^{2} \Rightarrow A\left(\xi^{1}\right)=1 .
\end{aligned}
$$

A partir de 31) e 32):

$$
\begin{array}{rlr}
\tau & =-\frac{c}{\alpha} \ln \left(-\alpha u / c^{2}\right) \quad \text { ou } \\
\tau & =\frac{c}{\alpha} \ln \left(\alpha v / c^{2}\right),
\end{array}
$$

como $\tilde{u}=\tilde{v}=c \tau$

$$
\begin{aligned}
\tilde{u} & =-\frac{c^{2}}{\alpha} \ln \left(-\alpha u / c^{2}\right) \Rightarrow u=-\frac{c^{2}}{\alpha} e^{-\alpha \tilde{u} / c^{2}} \\
\tilde{v} & =\frac{c^{2}}{\alpha} \ln \left(\alpha v / c^{2}\right) \Rightarrow v=\frac{c^{2}}{\alpha} e^{\alpha \tilde{v} / c^{2}}
\end{aligned}
$$

Embora tenhamos chegado nas expressões acima para a situação de um referencial comóvel ao observador acelerado, elas permanecem válidas para o caso não-comóvel $\left(d \xi^{1} \neq 0\right)$ já que envolvem coordenadas do cone de luz

$$
d s^{2}=e^{2 \alpha \xi^{1} / c^{2}}\left[\left(d \xi^{0}\right)^{2}-\left(d \xi^{1}\right)^{2}\right],
$$

e, finalmente, usando 39 e 40

$$
\begin{aligned}
x\left(\xi^{0}, \xi^{1}\right) & =c^{2} \frac{e^{\alpha \xi^{1} / c^{2}}}{\alpha} \cosh \left(\alpha \xi^{0} / c^{2}\right), \\
t\left(\xi^{0}, \xi^{1}\right) & =c \frac{e^{\alpha \xi^{1} / c^{2}}}{\alpha} \sinh \left(\alpha \xi^{0} / c^{2}\right) .
\end{aligned}
$$

Estas equações relacionam as coordenadas de um observador inercial com as coordenadas de um observador acelerado.

\subsection{Comparação entre o movimento relativístico de Rindler e MRUV da mecânica clássica}

Na mecânica clássica a equação de movimento de uma partícula sujeita a uma aceleração uniforme é descrita por

$$
\frac{d^{2} x}{d t^{2}}=a
$$

o que implica na seguinte equação de movimento

$$
x(t)=x_{0}+v_{0} t+\frac{1}{2} a t^{2} .
$$

Esta corresponde a uma parábola.

Podemos escolher convenientemente a origem do tempo de tal forma que em $t=-\infty$ a partícula esteja em $x=+\infty$ e, à medida que o tempo passa a partícula se aproxima da origem $(x=0)$ e, se supusermos que a aceleração aponta no sentido de $\mathrm{x}$ crescente, quanto mais a partícula se aproxima da origem, sua velocidade fica cada vez menor até parar em um ponto $x=x_{0}$ (em $t=0)$ e começar a retornar ao infinito aceleradamente. Assumindo o limite de baixas velocidades e para pequenos valores de $t$, é possível procedermos analogamente ao mostrado em [14, expandindo a equação (5) e 19 em séries de Taylor até primeira ordem:

$$
t=\tau\left(1+\frac{1}{2}(v / c)^{2}\right) \Rightarrow t \approx \tau
$$

$$
\begin{aligned}
x(t) & =\frac{c^{2}}{\alpha}\left(\frac{\exp (\alpha t / c)+\exp (-\alpha t / c)}{2}\right) \\
& =\frac{c^{2}}{2 \alpha}\left(1+\frac{\alpha t}{c}+\frac{1}{2 !}\left(\frac{\alpha t}{c}\right)^{2}+1-\frac{\alpha t}{c}\right. \\
& \left.+\frac{1}{2 !}\left(\frac{\alpha t}{c}\right)^{2}+\mathcal{O}\left((\alpha t / c)^{4}\right)\right) \\
& \approx \frac{c^{2}}{\alpha}+\frac{\alpha}{2} t^{2} .
\end{aligned}
$$

Comparando 46 com 44, é possível estabelecer a relação entre ambas escolhendo a aceleração constante $a=\alpha$ e $x_{0}=c^{2} / \alpha$, haja vista que já foi assumido anteriormente $v_{0}=0$.

Um gráfico representando este movimento é mostrado na Figura (4) onde os eixos foram rotacionados de $90^{\circ}$ para compararmos com o diagrama do espaço-tempo de Rindler. Nela, percebe-se que, quanto mais o módulo do tempo aumenta, mais o gráfico da parábola se afasta das retas $x=c t$ e $x=-c t$, ou seja, para valores grandes de $t$, pequenas variações temporais correspondem a grandes variações espaciais, dito de outra forma, a velocidade da partícula torna-se muito grande, isso porque derivando a equação 46 em relação ao tempo obtém-se

$$
\frac{d x}{d t}=v=\alpha t
$$


Desta expressão, vemos que quando o tempo tende ao infinito, a velocidade também torna-se infinitamente grande, logo, não há um valor limite para a velocidade na mecânica clássica.

Já para o caso do espaço-tempo de Rindler, o diagrama espaço-temporal possui a forma ilustrada na Figura (3) e as linhas de mundo destes observadores (com 4-aceleração uniforme) são hipérboles.

Aqui, vê-se que, quanto maior for o valor da coordenada tipo-tempo, mais a linha-mundo se aproximará das retas definidas por $x=t$ e $x=-t$, entretanto, sem alcançálas em um tempo finito. São portanto assíntotas que atuam como um horizonte para tais observadores. Deve haver assim um limite superior para a velocidade de observadores acelerados. Vejamos isto matematicamente. Analogamente ao que foi feito acima, tomemos a equação de movimento para um observador de Rindler dada pela equação 15 e derivemo-na com relação ao tempo

$$
\begin{aligned}
& x=\sqrt{\left(c^{2} / \alpha\right)^{2}+(c t)^{2}} \Rightarrow \frac{d x}{d t}=v=\frac{\alpha t}{\sqrt{1+\frac{\alpha^{2} t^{2}}{c^{2}}}}, \\
& v=\frac{c}{\sqrt{1+(c / \alpha t)^{2}}} .
\end{aligned}
$$

Quando $t \rightarrow \infty$ a velocidade tende ao valor $v \rightarrow c$. Assim, a velocidade da luz é a velocidade limite para qualquer observador acelerado.

De tudo o que discutimos sobre observadores uniforme-

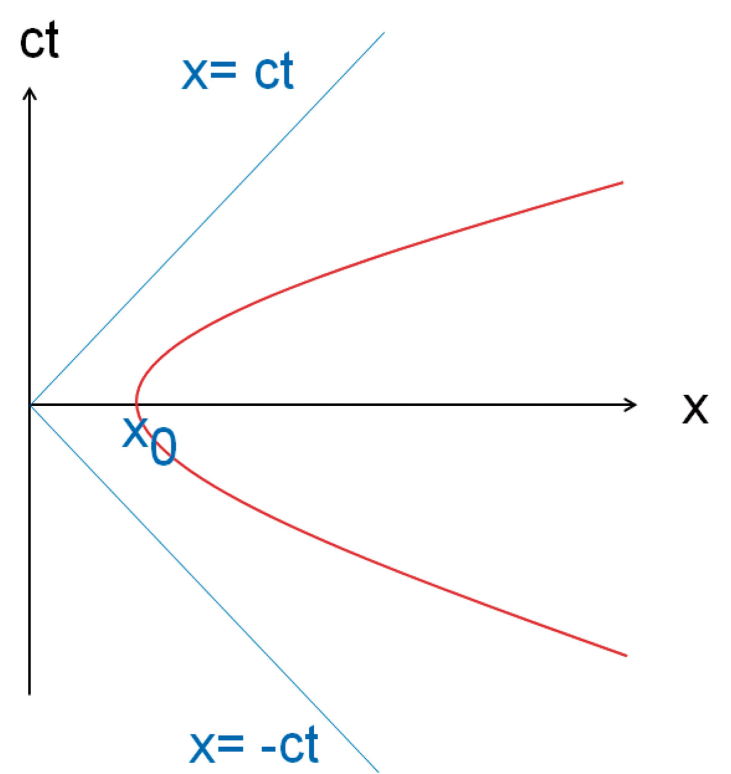

Figura 4: Diagrama para um observador em movimento retilíneo uniformemente acelerado na mecânica clássica sujeito a uma aceleração apontando no sentido positivo de $x$. Note que o tempo foi escolhido convenientemente, de modo que em $t=0$ a partícula esteja na posição de maior aproximação. Além disso, os eixos foram rotacionados de $90^{\circ}$ a fim de facilitar a comparação com o diagrama de Rindler. mente acelerados clássicos e observadores relativísticos uniformemente acelerados no espaço-tempo de Minkowski, percebemos que embora ambos sejam uniformemente acelerados há diferenças sensíveis. A primeira é que, no caso clássico a aceleração é composta totalmente por derivadas segundas das componentes espaciais com relação ao tempo ao passo que no caso uniformemente acelerado relativístico não são as componentes espaciais da aceleração que permanecem constante mas sim o módulo da 4-aceleração que inclui a parte tipo-espaço e a parte tipotempo. Depois, a velocidade no caso clássico pode aumentar indefinidamente. Já para o movimento relativístico a velocidade limite é a velocidade da luz. Finalmente, os diagramas de espaço-tempo para ambos diferem já que para o MRUV a trajetória é uma parábola enquanto que para os observadores de Rindler trata-se de hipérboles.

\section{Observadores uniformemente acelerados em um espaço-tempo em expansão}

Na primeira parte do presente trabalho, dedicamos nossa atenção ao estudo do movimento de observadores relativísticos uniformemente acelerados no espaço-tempo de Minkowski. Agora, buscaremos estender a noção de observadores uniformemente acelerados para um espaço-tempo em expansão, salientando as semelhanças, diferenças e dificuldades em relação ao caso plano e estático.

Como métrica de fundo, utilizaremos a de FriedmannLemaître-Robertson-Walker (FLRW) espacialmente plana $(k=0)$ 15]:

$$
d s^{2}=c^{2} d t^{2}-R^{2}(t)\left[d r^{2}+d \Omega^{2}\right],
$$

onde $R(t)$ é o fator de expansão e $d^{2} \Omega=d \theta^{2}+\sin ^{2} \theta d \phi^{2}$. Para nossos objetivos, nos restringiremos ao caso radial, isto é, $d \Omega=0$.

Lembremo-nos agora que, como estamos trabalhando em um espaço-tempo curvo, derivadas ordinárias devem ser substituídas por derivadas covariantes. Isto é importante pois, embora ainda tenhamos a condição

$$
g_{\mu \nu} a^{\mu} a^{\nu}=-\alpha^{2}=c t e,
$$

as componentes da 4-aceleração não serão somente $t^{\prime \prime} \mathrm{e}$ $r^{\prime \prime}$, a exemplo do que fizemos no caso plano, mas deve-se considerar a expressão completa:

$$
a^{\mu}=\frac{d^{2} x^{\mu}}{d \tau^{2}}+\Gamma_{\alpha \beta}^{\mu} \frac{d x^{\alpha}}{d \tau} \frac{d x^{\beta}}{d \tau},
$$

onde

$$
\Gamma_{\alpha \beta}^{\mu}=\frac{1}{2} g^{\mu \lambda}\left(g_{\lambda \alpha, \beta}+g_{\beta \lambda, \alpha}-g_{\alpha \beta, \lambda}\right),
$$

são os símbolos de Christoffel 7, 12 .

Aqui, as "vírgulas" representam derivadas ordinárias com 
relação ao parâmetro seguinte.

Para a métrica de FLRW radial, somente os símbolos

$$
\begin{aligned}
& \Gamma^{0}{ }_{11}=-g^{00} g_{11,0}=\frac{R}{c} \frac{d R}{d t}, \\
& \Gamma_{01}^{1}=\Gamma_{10}^{1}=g^{11} g_{11,0}=\frac{1}{R c} \frac{d R}{d t} \equiv \frac{H(t)}{c},
\end{aligned}
$$

são não-nulos. $H(t)$ é o parâmetro de Hubble, que contêm informações sobre a taxa de expansão do universo.

Com base nisto, a equação (50) fornece

$$
\begin{aligned}
& \left(a^{0}\right)^{2}-R^{2}\left(a^{1}\right)^{2}=-\alpha^{2}, \\
& \left(c^{2} t^{\prime \prime}+R^{2} H(t) r^{\prime 2}\right)^{2}-R^{2}\left(r^{\prime \prime}+2 H(t) r^{\prime} t^{\prime}\right)^{2} \\
& =-\alpha^{2} .
\end{aligned}
$$

Entretanto, somente (55) não nos permite obter a equação de movimento já que não sabemos nem $r$, nem $t$. A outra equação vem do elemento de linha:

$$
d s^{2}=c^{2} d \tau^{2}=c^{2} d t^{2}-R^{2}(t) d r^{2}
$$

$$
\begin{aligned}
\left(t^{\prime}\right)^{2}-\frac{R^{2}(t)}{c^{2}}\left(r^{\prime}\right)^{2}=1 \\
\left(r^{\prime}\right)^{2}=\frac{c^{2}\left[\left(t^{\prime}\right)^{2}-1\right]}{R^{2}} \\
\Rightarrow \frac{r^{\prime \prime}}{r^{\prime}}=\frac{t^{\prime \prime} t^{\prime}}{t^{\prime 2}-1}-H t^{\prime} .
\end{aligned}
$$

Substituindo 56 em 55

$$
\begin{aligned}
& t^{\prime 2}\left(t^{\prime 2}-1\right)\left[\frac{t^{\prime \prime}}{\left(t^{\prime 2}-1\right)}+H(t)\right]^{2} \\
& -\left[t^{\prime \prime}+H(t)\left(t^{\prime 2}-1\right)\right]^{2}=\frac{\alpha^{2}}{c^{2}} .
\end{aligned}
$$

Esta equação diferencial pode ser simplificada utilizando novamente a mudança de variável $t^{\prime}=\cosh z$.

$$
\begin{aligned}
\cosh ^{2} z \sinh ^{2} z\left[\frac{z^{\prime} \sinh z}{\sinh ^{2} z}+H(t)\right]^{2}-\left[z^{\prime} \sinh z+H(t) \sinh ^{2} z\right]^{2} & =\frac{\alpha^{2}}{c^{2}} \\
z^{\prime 2}+2 z^{\prime} H(t) \sinh z+H^{2}(t) \sinh ^{2} z & =\frac{\alpha^{2}}{c^{2}} \\
\left(z^{\prime}+H(t) \sinh z\right)^{2} & =\frac{\alpha^{2}}{c^{2}} \\
z^{\prime}+H(t) \sinh z & =\frac{\alpha^{2}}{c^{2}}
\end{aligned}
$$

Mas como $t^{\prime}=\cosh z, t^{\prime \prime}=z^{\prime} \sinh z \Rightarrow z^{\prime}=t^{\prime \prime} / \sqrt{t^{\prime 2}-1}$ e ficamos com a seguinte equação diferencial

$$
t^{\prime \prime}+H(t)\left[t^{\prime 2}-1\right]-\frac{\alpha}{c} \sqrt{t^{\prime 2}-1}=0
$$

Que, juntamente com (56), descrevem o movimento de um observador acelerado no espaço-tempo de FLRW.

A resolução de (58) depende do modelo cosmológico e será objeto de estudo em um trabalho futuro.

\section{Conclusão}

Neste trabalho apresentamos, primeiramente, as principais características associadas ao espaço-tempo de Rindler, desenvolvendo equações e passagens com detalhes, aproveitamos ainda para comparar o comportamento de observadores uniformemete acelerados nos casos clássico e relativístico: enquanto que a linha de mundo de um observador clássico em MRUV é descrito no diagrama de espaço-tempo por uma parábola, o observador relativístico, também acelerado, se move ao longo de uma linha de mundo hiperbólica, tendo como assíntotas as retas $\mathrm{ct}=\mathrm{x}$ e ct $=-\mathrm{x}$, geradoras do cone de luz, o que limita sua rapidez à velocidade da luz e atua como horizontes. Por fim, abordou-se o caso de observadores uniformemente acelerados no espaço-tempo em expansão de Friedman-Lemaître-Robertson-Walker.

A presença de um fator de expansão conduziu a equações diferenciais muito semelhantes àquelas do espaço-tempo de Rindler, apenas com um termo adicional, proporcional ao parâmetro de Hubble, $H(t)$, o que nos permite vislumbrar implicações cosmológicas a serem abordadas futuramente.

\section{Referências}

[1] W. Rindler, American Journal of Physics 34, 1174 (1966). 
[2] S.A. Fulling, Phys. Rev. D. 7, 2850 (1973).

[3] P.C.W. Davies, Journ. of Phys. A. 8, 609 (1975).

[4] W.G. Unruh, Phys. Rev. D. 14, 870 (1976).

[5] G.E. Matsas, Rev. Bras. Ens. Fís. 27, 137 (2005).

[6] W. Rindler, Relativity (Oxford University Press, New York, 2006).

[7] R.M. Wald, General Relativity (The University of Chicago Press, Chicago, 1984).

[8] S.M. Carroll, Spacetime and Geometry: An Introduction to General Relativity (Addison Wesley, 2004).

[9] B. Shultz, A First Course in General Relativity (Cambridge University Press, New York, 2009).

[10] I. Newton, Principia: Princípios Matemáticos de Filosofia Natural, (EDUSP, São Paulo, 1990), v. 1. p. 6-7.

[11] C.W. Misner, K.S. Thorne and J.A. Wheeler, Gravitation (Freeman, San Francisco, 1973).

[12] R. D'Inverno, Introducing Einstein's Relativity (Clarendon Press, New York, 1992).

[13] L. Landau and E.L. Lifshitz, The Classical Theory of Fields (Pergamon Press, Oxford, 1976).

[14] G.F.T. Castillo, Revista Mexicana de Física 52, 70 (2006).

[15] V. Mukhanov, Physical Foundations of Cosmology (Cambridge University Press, New York, 2005). 